\title{
No relation between body mass and gastro-oesophageal reflux symptoms in a Swedish population based study
}

\author{
J Lagergren, R Bergström, O Nyrén
}

\begin{abstract}
Background-There is a widespread notion that obesity leads to gastrooesophageal reflux but scientific evidence of an association is limited and inconsistent.

Aims-To estimate the strength of the association between body mass and reflux symptoms, we performed a population based cross sectional interview study.

Subjects-Population based, randomly selected, middle aged or elderly persons in Sweden in 1995-1997.

Methods-At face-to-face interviews we asked a stratified sample of Swedes about body measures and occurrence of reflux symptoms. Odds ratios (OR) with $95 \%$ confidence intervals (CI), calculated by logistic regression with multivariate adjustments for covariates, were the measures of association.

Results-Reflux symptoms occurring at least once a week more than five years before the interview were reported by 135 $(16 \%)$ of the 820 interviewees. Among those who had ever been overweight during adulthood (body mass index (BMI) $\geqslant 25 \mathrm{~kg} / \mathrm{m}^{2}$ ), the $\mathrm{OR}$ of having recurrent reflux symptoms was 0.99 (95\% CI $0.66-$ 1.47) compared with those who were never overweight. There was no association between BMI at age 20, BMI 20 years before the interview, or maximum adult BMI and occurrence of reflux symptoms: ORs per unit increase in BMI were 1.00 (95\% CI 0.93-1.09), 1.03 (95\% CI 0.96$1.10)$, and $1.01(95 \% \mathrm{CI}=0.951 .07)$, respectively. There was no association between BMI and severity or duration of reflux symptoms.
\end{abstract}

Conclusions-Gastro-oesophageal reflux symptoms occur independently of body mass index. Weight reduction may not be justifiable as an antireflux therapy. (Gut 2000;47:26-29)

Keywords: gastro-oesophageal reflux; body mass index; obesity; population based study

Statistics, Uppsala

University, Uppsala,

Sweden

R Bergström

Correspondence to:

J Lagergren, Division of

Surgery, Karolinska

Institutet, Danderyd

Hospital, SE-182 88

Danderyd, Sweden. Email

Jesper.Lagergren@mep.ki.se

Accepted for publication

21 December 1999 and and obese patients who seek medical care for symptoms suggestive of reflux are often recommended by clinicians to reduce their body weight to relieve the symptoms. However, the scientific basis of a positive association between reflux and overweight is surprisingly weak. To our knowledge there are no published investigations in which a rigorous epidemiological approach has been used to address this question. In two previous studies a positive association was found between body weight and endoscopically detected reflux oesophagitis, ${ }^{12}$ and in two studies there was a moderately strong association between reflux symptoms and obesity. ${ }^{34}$ In one study in which reflux was assessed by $\mathrm{pH}$ measurements, no difference in the occurrence of reflux was observed between massively obese patients and control subjects of normal weight. ${ }^{5}$ Because of the preconceptions among doctors, overweight may be linked to the likelihood that a patient with reflux symptoms is referred for endoscopy or $\mathrm{pH}$ monitoring. Therefore, an epidemiological approach among non-patients is preferable.

It is of obvious clinical importance to establish if there is an association between body mass and gastro-oesophageal reflux. A true strong positive association would strengthen the role of weight reduction as an important part of antireflux treatment in overweight patients while such a strategy is likely to be unsuccessful if the association is weak or absent. Heartburn and regurgitation are considered reasonably accurate markers of gastrooesophageal reflux disease. ${ }^{6-10}$ We investigated the association between symptoms of reflux and body mass index (BMI) in a population based study, with adjustment for suspected confounding variables.

\section{Methods}

DESIGN

We have conducted a nationwide case control study in Sweden with the primary aim of identifying risk factors for adenocarcinoma of the oesophagus and gastric cardia. ${ }^{11}$ From this case control study we selected the population based control subjects for the present cross sectional analysis. Subjects were randomly selected from 10 year age and sex strata in the entire Swedish population using the continuously updated computerised population register, and were frequency matched so that their age and sex distributions resembled those of the cancer cases. Subjects were less than 80 years of age, were born in Sweden, and were living in this country during the study period (1 December 1994 to 31 December 1997).
Abbreviations used in this paper: $\mathrm{BMI}$, body mass index; OR, odds ratio; CI, confidence interval. 
DATA COLLECTION

All subjects underwent computer aided faceto-face interviews by specially trained professional interviewers from Statistics Sweden. We collected information on average adult height, maximum adult weight, weight at age 20 , and weight 20 years before the interview. Body mass index (BMI), a validated measure of body mass independent of height, ${ }^{12}$ was calculated as body weight divided by the square of body height in metres $\left(\mathrm{kg} / \mathrm{m}^{2}\right)$. Furthermore, subjects were asked about their lifetime history of recurrent heartburn or regurgitation which are the main symptoms of reflux, ${ }^{6}$ but symptoms occurring during the most recent five years before the interview were not included. Reflux was defined as recurrent heartburn or regurgitation occurring at least once a week for a period of no less than one year. Those who reported recurrent reflux symptoms were questioned about symptom duration (number of years), symptom frequency (number of episodes per year/month/week/day), and timing of symptoms (nightly occurrence or not). Finally, we collected detailed information on potential confounding factors which were categorised as follows: age (in five year groups), sex (male/ female), tobacco smoking (non-smokers, former smokers, and current smokers of cigarettes, cigars, and pipes), alcohol use (grams of pure alcohol per week with respondents categorised into four classes), meal sizes (with respondents categorised into three classes), and physical activity during leisure time and at work (with respondents categorised into four classes).

\section{STATISTICAL ANALYSES}

The relation between the explanatory BMI variables and the dependent dichotomous reflux variable was modelled using logistic regression, estimated by the maximum likelihood method. ${ }^{13}$ The basic beta parameters $(\beta)$ of this model and their standard errors (SE) were converted into odds ratios (OR) and 95\% confidence intervals (CI). In analyses of categorised BMI data, the cut off points were based on the WHO classification of overweight and obesity. ${ }^{14} \mathrm{~A}$ BMI value $\geqslant 25-30$ represents overweight and BMI $>30$ indicates obesity. Thus a BMI value $<25$ is regarded as normal. For quantitative dependent variables such as duration of reflux symptoms, we used linear regression estimated by ordinary least squares. To allow for non-linear relations, BMI was divided into classes and each class was entered into the model as a dummy variable ( 1 or 0 ). Hence the parameter shows to what extent individuals in the higher BMI category on average differ from individuals in the reference category (BMI <25) with regard to the dependent variable. In the baseline model, adjustments were made for age and sex, while in the multivariate analyses we adjusted for all six covariates listed above.

ETHICS

The study was approved by all regional ethics committees in Sweden. Informed consent was
Table 1 Characteristics of subjects by reflux symptom status

\begin{tabular}{lcc}
\hline & \multicolumn{2}{c}{$\begin{array}{l}\text { Reflux symptoms at least once } \\
\text { a week for 1 y }\end{array}$} \\
\cline { 2 - 3 } & Yes more & No \\
\hline $\mathrm{n}$ & 135 & 685 \\
Median age (y) & 70 & 68 \\
Mean age (y) & 66 & 66 \\
No of males (\%) & $118(87 \%)$ & $561(82 \%)$ \\
Ever tobacco smokers & $83(61 \%)$ & $412(60 \%)$ \\
High alcohol consumptiont & $26(19 \%)$ & $152(22 \%)$ \\
\hline
\end{tabular}

*Tobacco smoking status included cigarette, cigar, and pipe smoking and the status two years before the interview was assessed.

†High alcohol consumption was defined as more than $70 \mathrm{~g}$ of pure alcohol per week.

obtained from all participants prior to the interview.

\section{Results}

Among the selected 1128 population based subjects, $820(73 \%)$ participated in the interview. Non-participation was due to unwillingness in $210(19 \%)$, physical or mental disorders prohibiting an interview in $70(6 \%)$, and incorrect addresses in $28(2 \%)$. The interviewers deemed the quality of the answers about reflux symptoms and body measures to be uncertain in three $(0.4 \%)$ and $15(2 \%)$ respondents, respectively. Subject characteristics of the 135 $(16 \%)$ who reported recurrent reflux symptoms were compared with those of the 685 individuals who did not. There were no differences in the frequency of tobacco smoking or alcohol consumption between these groups, and age and sex distributions were similar (table 1).

We analysed maximum adult BMI, BMI 20 years before the interview, and BMI at age 20 as explanatory variables. As true obesity was rare at age 20 and 20 years before the interview, we combined overweight and obesity in the analyses of these two BMI variables. We found no statistically significant associations between overweight and/or obesity and gastrooesophageal reflux in the analyses of any of the three BMI variables. All point estimates were close to unity (table 2). Among those who had ever been overweight or obese (BMI $\geqslant 25)$, the adjusted OR was 0.99 (95\% CI $0.66-1.47$ ) compared with those who had never been overweight. Analyses with the BMI variables in continuous forms confirmed the absence of any tendency towards an association (table 2). Multivariate adjustments for potential confounding factors did not substantially alter any of the age or sex adjusted estimates (table 2).

To determine if increasing severity of reflux symptoms was associated with BMI, we constructed a symptom severity score. ${ }^{11}$ This score included: (1) symptom characteristics (heartburn only, 1 point; regurgitation only, 1 point; heartburn and regurgitation combined 1.5 points); (2) occurrence of nightly symptoms (yes, 2 points; no, 0 points); and (3) symptom frequency (once a week, 0 points; $2-6$ times/week, 1 point; 7-15 times/week, 2 points; $>15$ times/week, 3 points). These points were summed to produce a total symptom score for each of the 135 individuals with recurrent 
Table 2 Association of body mass index (BMI)* at the time of maximum adult weight, 20 years before the interview, and at age 20, with risk of gastro-oesophageal refluxt. Age and sex adjusted, and multivariately $\ddagger$ adjusted odds ratios (OR) and $95 \%$ confidence intervals (CI)

\begin{tabular}{|c|c|c|c|}
\hline & $\begin{array}{l}\text { No with/ } \\
\text { without reflux }\end{array}$ & $\begin{array}{l}\text { Age and sex adjusted } \\
\text { OR }(95 \% \text { CI })\end{array}$ & $\begin{array}{l}\text { Multivariately } \\
\text { adjusted OR } \\
(95 \% \text { CI) }\end{array}$ \\
\hline \multicolumn{4}{|l|}{ Maximum BMI } \\
\hline BMI $<25$ & $50 / 250$ & 1.00 (reference) & 1.00 (reference) \\
\hline BMI 25-30 (overweight) & $63 / 328$ & $0.94(0.62-1.42)$ & $0.95(0.62-1.44)$ \\
\hline BMI $>30$ (obesity) & $22 / 103$ & $1.06(0.61-1.86)$ & $1.13(0.64-2.01)$ \\
\hline $\mathrm{BMI}$ as continuous variable & $135 / 685$ & $1.00(0.95-1.06) \$$ & $1.01(0.95-1.07) \S$ \\
\hline \multicolumn{4}{|l|}{ BMI 20 years before interview } \\
\hline BMI $<25$ & $94 / 479$ & 1.00 (reference) & 1.00 (reference) \\
\hline $\mathrm{BMI} \geqslant 25$ (overweight or obesity) & $41 / 202$ & $0.98(0.65-1.49)$ & $1.01(0.67-1.54)$ \\
\hline $\mathrm{BMI}$ as continuous variable & $135 / 685$ & $1.02(0.95-1.09) \$$ & $1.03(0.96-1.10)$ \\
\hline \multicolumn{4}{|l|}{$\mathrm{BMI}$ at age 20} \\
\hline $\mathrm{BMI}<25$ & $125 / 598$ & 1.00 (reference) & 1.00 (reference) \\
\hline $\mathrm{BMI} \geqslant 25$ (overweight or obesity) & $10 / 83$ & $0.55(0.27-1.09)$ & $0.54(0.27-1.09)$ \\
\hline $\mathrm{BMI}$ as continuous variable & $135 / 685$ & $1.00(0.93-1.08) \S$ & $1.00(0.93-1.09) \S$ \\
\hline
\end{tabular}

${ }^{\star}$ Body mass index was calculated as body weight divided by the square of body height in metres $\left(\mathrm{kg} / \mathrm{m}^{2}\right)$.

†Gastro-oesophageal reflux was defined as recurrent and regular heartburn and/or regurgitation occurring at least once a week.

$\ddagger$ Adjustments were made for age, sex, tobacco smoking, alcohol use, meal sizes, and physical activity.

jOdds ratio per unit increase in BMI.

Table 3 Association between body mass index (BMI)* levels at three different time points in life and severity of reflux symptomst analysed in continuous form among 135 individuals with reflux symptoms

\begin{tabular}{|c|c|c|c|c|c|c|}
\hline & \multicolumn{3}{|c|}{ Age and sex adjusted } & \multicolumn{3}{|c|}{ Multivariately $\neq$ adjusted } \\
\hline & $\beta$ & $S E(\beta)$ & $p$ & $\beta$ & $S E(\beta)$ & $p$ \\
\hline \multicolumn{7}{|l|}{ Maximum adult BMI } \\
\hline $\mathrm{BMI}<25$ & \multicolumn{3}{|c|}{0.0 (reference) } & \multicolumn{3}{|c|}{0.0 (reference) } \\
\hline BMI 25-30 (overweight) & -0.06 & 0.10 & 0.58 & -0.06 & 0.11 & 0.56 \\
\hline BMI > 30 (obesity) & -0.03 & 0.14 & 0.84 & -0.02 & 0.15 & 0.90 \\
\hline \multicolumn{7}{|l|}{ BMI 20 years before interview } \\
\hline BMI $<25$ & \multicolumn{3}{|c|}{0.0 (reference) } & \multicolumn{3}{|c|}{0.0 (reference) } \\
\hline $\begin{array}{l}\text { BMI } \geqslant 25 \text { (overweight or } \\
\text { obesity) }\end{array}$ & -0.05 & 0.10 & 0.62 & -0.05 & 0.11 & 0.65 \\
\hline \multicolumn{7}{|l|}{ BMI at age 20} \\
\hline $\mathrm{BMI}<25$ & \multicolumn{3}{|c|}{0.0 (reference) } & \multicolumn{2}{|c|}{0.0 (reference) } & \\
\hline $\begin{array}{l}\text { BMI } \geqslant 25 \text { (overweight or } \\
\text { obesity) }\end{array}$ & -0.27 & 0.15 & 0.07 & -0.27 & 0.15 & 0.07 \\
\hline \multicolumn{7}{|c|}{$\begin{array}{l}\text { ^Body mass index was calculated as body weight divided by the square of body height in metres } \\
\left(\mathrm{kg} / \mathrm{m}^{2}\right) \text {. } \\
+ \text { Severity of gastro-oesophageal reflux was defined according to a reflux score The score included } \\
\text { symptom characteristics (heartburn only, } 1 \text { point; regurgitation only, } 1 \text { point; heartburn and } \\
\text { regurgitation combined, } 1.5 \text { points), nightly symptoms (yes, } 2 \text { points), and symptom frequency } \\
\text { (once a week, } 0 \text { points; } 2-6 \text { times/week, } 1 \text { point; } 7-16 \text { times/week, } 2 \text { points; }>16 \text { times/week, } \\
3 \text { points). } \\
\ddagger \text { Adjustments were made for age, sex, tobacco smoking, alcohol use, meal sizes, and physical } \\
\text { activity. }\end{array}$} \\
\hline
\end{tabular}

symptoms of reflux. The sum, ranging from 1 to 6.5 points, was the dependent variable in linear regression models restricted to the 135 individuals with reflux symptoms. No significant associations or trends were found between overweight or obesity and increasing severity scores (table 3 ). This lack of association was evident for all three BMI variables. Multivariate adjustments did not change the $\beta$ parameter estimates. Hence the symptoms in those with a high BMI were no worse than those in lighter subjects. Moreover, there was no significant association between BMI and reflux symptom duration (data not shown).

\section{Discussion}

Our population based cross sectional study revealed no evidence of an association between $\mathrm{BMI}$ and gastro-oesophageal reflux symptoms. Compared with the leanest, obese subjects had no greater propensity for reflux symptoms. Nor did they have more severe reflux or a longer reflux duration than thinner subjects.
Our findings are consistent with the results of two previous studies in massively obese subjects; one showed no differences in oesophageal $\mathrm{pH}$ compared with normal weight subjects ${ }^{5}$ and the other found no improvement in reflux symptoms or oesophageal $\mathrm{pH}$ after weight reduction. ${ }^{15}$ Our results are in conflict with two case series consisting of patients referred for gastroscopy. ${ }^{12}$ In these investigations the average BMI was significantly higher among patients with reflux oesophagitis than in those without. However, given the widespread view of a link between BMI and reflux, doctors may have perceived the symptoms as a natural consequence of obesity. Therefore, obese subjects may first have been recommended to decrease their body weight while thinner subjects could have been referred for gastroscopy more readily and included in the studies. Hence, for selection for endoscopy, obese patients may have had more severe reflux symptoms and a higher prevalence of oesophagitis compared with thinner subjects. Detection bias on the part of the endoscopists is also a possibility. In two studies in uninvestigated subjects there was a positive association between obesity and the occurrence of reflux symptoms ${ }^{34}$ but no adjustments were made for potential confounding factors. It is possible that the positive associations previously reported were simply the results of confounding.

There is no generally agreed standard on how to define or measure the occurrence of gastro-oesophageal reflux disease. Both endoscopy and 24 hour $\mathrm{pH}$ monitoring have significant limitations. Only about one third of patients with reflux disease have endoscopically detectable signs of reflux. ${ }^{16}$ The specificity of endoscopic low grade oesophagitis may also be less than ideal. $\mathrm{pH}$ measurements in nonoesophagitis cases are limited by ambiguity with regard to appropriate cut off points between disease and normality, in turn due to lack of a gold standard comparison in these cases. ${ }^{17}$ As reflux symptoms are considered to be reliable and valid indices of gastrooesophageal reflux disease, ${ }^{6-10}$ analysis of the main symptoms, heartburn and regurgitation, is probably the most useful method for diagnosing this condition. ${ }^{16}$ However, misclassification of this condition might have driven our measures of association spuriously towards the null. However, lack of association in any of our analyses, including those of symptom severity, argues against an important relation.

There is a risk of under reporting of reflux symptoms in our study if the use of antireflux medication concealed the symptoms effectively. Among the study subjects, however, all who reported regular use of antireflux medication also reported reflux symptoms occurring often enough to be included among the reflux patients. In the adult US population, the proportion of individuals with reflux symptoms occurring at least once a week has been estimated to be $15-20 \% .^{18}{ }^{19}$ This value is in agreement with our study, where the corresponding proportion was $16 \%$. This conformity seems to indicate good validity of our reflux symptom data and that our randomly selected 
subjects are well representative of the population at large.

In a cross sectional analysis, the possibility of reversed causality (weight reduction because of obesity) needs to be considered. Subjects with obesity induced reflux may have been recommended by doctors or lay friends to lose weight. Furthermore, the presence of severe symptoms could theoretically have led to involuntary caloric restrictions. However, the availability of information on BMI at several points in time, including at age 20, and the absence of association with occurrence of reflux for any of these BMI values, allay our concerns that a true association with reflux was cancelled by differential weight reduction.

The subjects in our study were frequency matched for age and sex to patients with oesophageal cancer in the nationwide case control study. ${ }^{11}$ Therefore, our subjects were recruited mainly from the male Swedish population and predominantly from older age strata. As they were randomly selected from the strata, well defined owing to the continuously updated Swedish population register, the internal validity of our study should not have been affected by this restriction with regard to age and sex. However, inference to other populations than men above 50 years should be drawn with some caution.

It is unlikely that we had a differential loss of obese persons with reflux symptoms willing to undergo the interview. Nevertheless, with a non-participation rate of $27 \%$ it was important to identify potential selection bias. We conducted a separate analysis of a group of 24 control subjects who had refused participation but at a later stage were persuaded to enter the study. This small group could be considered a sample group of the non-participating control subjects. Although lacking in precision, this analysis revealed that the distributions of BMI (at all three time points analysed), reflux symptoms, and all of the confounding variables were strikingly similar in the sample group compared with those in the total group (data not shown), indicating absence of important selection bias.

The confidence intervals of our estimates were relatively narrow, and the point estimates were reassuringly close to unity in all separate analyses. Furthermore, no dose-response association was found between overweight and increasing severity or duration of reflux symptoms. Hence chance is an unlikely explanation for the lack of association found in this study.

Our data were adjusted for age, sex, smoking, alcohol use, meal sizes, and physical activity. The age and sex adjusted estimates remained almost unchanged after inclusion of the other four covariates in the multivariate analysis, indicating that there was no confounding of importance from these factors.

In view of recent revelations of serious long term consequences of symptomatic reflux disease ${ }^{11}$ there is a need for simple methods for preventing reflux. Although our data seem to indicate that weight reduction might not lead to the desired reflux control, it may still be beneficial. It was recently shown that obesity per se is a strong risk factor for adenocarcinoma of the oesophagus and gastric cardia, independent of reflux. ${ }^{20}$ But weight reduction may not be an adequate substitute for medical or surgical treatment if control of symptoms is the primary goal.

Financial support: National Cancer Institute (NCI) grant R01 CA57947-03, Swedish Cancer Society (JL and ON), and Dalarna Research Institute (JL).

1 Stene-Larsen G, Weberg R, Froyshov Larsen I, et al. Relationship of overweight to hiatus hernia and reflux oesophagitis. Scand F Gastroenterol 1988;23:427-32.

2 Chang CS, Poon SK, Lien HC, et al. The incidence of reflux esophagitis among the Chinese. Am $f$ Gastroenterol 1997;92:668-71.

3 Isolauri J, Laippala P. Prevalence of symptoms suggestive of gastro-oesophageal reflux disease in an adult population. Ann Med 1995;27:67-70.

4 Romero Y, Cameron AJ, Locke GR III, et al. Familial aggregation of gastroesophageal reflux in patients with Barrett's gation of gastroesophageal reflux in patients with Barrett's ogy 1997;113:1449-56.

ogy 1997; 113:1449-56.
5 Lundell L, Ruth M, Sandberg N, et al. Does massive obesity promote abnormal gastroesophageal reflux? Dig Dis Sci 1995;40:1632-35.

6 Ter RB, Castell DO. Gastroesophageal reflux disease in patients with columnar-lined esophagus. Gastroenterol Clin North Am 1997;26:549-63.

7 Klauser AG, Schindlbeck NE, Müller-Lissner SA. Symptoms in gastro-oesophageal reflux disease. Lancet 1990;1: 205-8.

8 Locke GR, Talley NJ, Zinsmeister AR. A new questionnaire for gastroesophageal reflux disease. Mayo Clin Proc 1994;69:539-47.

9 Williford WO, Krol WF, Spechler SJ. Development for and results of the use of a gastroesophageal reflux disease activity index as an outcome variable in a clinical trial. VA Cooperative Study Group on gastroesophageal reflux disease (GERD). Control Clin Trials 1994;16:335-48.

10 Revicki DA, Wood M, Wiklund I, et al. Reliability and validity of the gastrointestinal symptom rating scale in patients with gastroesophageal reflux disease. Qual Life Res 1998;7: $75-83$.

11 Lagergren J, Bergström R, Lindgren A, et al. Symptomatic gastroesophageal reflux is a strong risk factor for esophageal adenocarcinoma. $N$ Engl f Med 1999;340:825-31.

12 Garrow JS, Webster Y. Quetelet's index (W/H2) as a measure of fatness. Int F Obes 1985;9:147-53.

13 Breslow NE, Day NE. Statistical methods in cancer research. Volume I-The analysis of case-control studies. IARC Sci Publ 1980;32:5-338.

14 Joint $\mathrm{FAO} / \mathrm{WHO} / \mathrm{UNU}$ Expert Consultation. Energy and protein requirements. WHO Technical Report Series. Geneva: protein requirements. WHO

15 Kjellin A, Ramel S, Rossner S, et al. Gastro-oesophageal reflux in obese patients is not reduced by weight reduction. reflux in obese patients is not reduced

16 Dent J. Gastro-oesophageal reflux disease. Digestion 1998; 59:433-45.

17 Richter JE. Ambulatory esophageal pH monitoring. Am $\mathcal{F}$ Med 1997;103:130-4S.

18 Kahrilas PJ. Gastroesophageal reflux disease. FAMA 1996; 276:983-8.

19 Locke GR, Talley NJ, Fett SL, et al. Prevalence and clinical spectrum of gastroesophageal reflux: a population-based study in Olmsted County, Minnesota. Gastroenterology 1997;112:1448-56.

20 Lagergren J, Bergström R, Nyrén O. Body mass as a risk factor for esophageal and gastric cardia adenocarcinoma. Ann Intern Med 1999;130:883-90. 